\title{
Tecnología móvil en el aprendizaje universitario
}

\author{
Mobile Technology in University Learning
}

\section{Tecnologia móvel na aprendizagem universitária}

\author{
Jorge Nelson Tejada Campos* id Israel Barrutia Barreto** \\ * Licenciado en Educación Matemática, Docente en Universidad Nacional de \\ Cajamarca. ** Superintendencia Nacional de Aduanas y Administración Tributaria \\ (SUNAT), Perú. Dirección electrónica: innovasciencia@gmail.com https://orcid. \\ org/0000-0002-5728-0651
}

\begin{abstract}
OPEN ACCESS
DOI: http://dx.doi.org/10.18634/sophiaj. 17v.1i.1016

Información del artículo

Recibido: octubre 08 de 2020

Revisado: enero 12 de 2021

Aceptado: abril 10 de 2021

Publicado: abril 15 de 2021

Palabras clave: Aprendizaje, aprendizaje móvil, educación, educación superior
\end{abstract} tecnología móvil.

Keywords: Mobile learning, mobile technology, learning, higher education, education.

Palavras-chave: Aprendizagem móvel, tecnologia móvel, aprendizagem, ensino superior, educação.

\section{Cómo citar: /how cite:}

Tejada, J.N., Barrutia, I. (2021) Tecnología móvil en el aprendizaje universitario. Sophia 17(1), e1016

Sophia-Educación, volumen 17 número 1. enero/junio 2021. Versión español

\section{RESUMEN}

En la última década han surgido un número significativo de iniciativas que apuntan a utilizar tecnologías y aplicaciones móviles con fines educativos, sin embargo, la implementación no ha sido totalmente efectiva debido a diversas dificultades. En este trabajo se exploraron las actitudes de los alumnos y docentes hacia el uso del aprendizaje móvil en instituciones de educación superior. Se tomaron muestras de estudiantes y docentes de cinco universidades de Cajamarca en Perú a los cuales se les aplicaron encuestas que fueron validadas por expertos y la confiabilidad fue evaluada mediante alfa de Cronbach. Se evaluó si existen diferencias estadísticamente significativas entre las actitudes de los estudiantes con relación a la edad, sexo, especialidad y tipo de móvil usado. Asimismo, se evaluó si existen diferencias estadísticamente significativas entre las actitudes de los docentes con relación a la edad, sexo, experiencia universitaria, nivel académico y tipo de móvil usado. Se realizaron pruebas de $t$ de Student y un análisis de varianza unidireccional (Anova) por cada indicador considerado. Los resultados revelaron que existen diferencias estadísticamente significativas entre las actitudes de los estudiantes con respecto al tipo de dispositivo móvil. La aparición de las tecnologías de aprendizaje móvil tuvo un impacto significativo en la tecnología educativa. 
Copyright 2021 Universidad La Gran Colombia

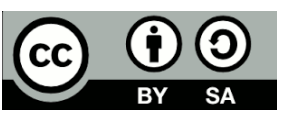

Conflicto de interés:

Los autores declaran no tener ningún conflicto de interés.

Correspondencia de autor:

jonetecampos@gmail.com israel20barrutia@gmail.com

\section{ABSTRACT}

In the last decade, a significant number of initiatives aimed at using mobile technologies and applications for educational purposes have emerged, however, implementation has not been fully effective due to various difficulties. This paper explored the attitudes of students and teachers towards the use of mobile learning in higher education institutions. Students and teachers from five universities in Cajamarca, Peru, were sampled and administered surveys that were validated by experts and whose reliability was evaluated using Cronbach's alpha. It was evaluated whether there are statistically significant differences between students' attitudes in relation to age, sex, major and type of cell phone used. Likewise, it was evaluated whether there are statistically significant differences between teachers' attitudes in relation to age, sex, university experience, academic level and type of cell phone used. Student's t-tests and a one-way analysis of variance (ANOVA) were performed for each indicator considered. The results revealed that there are statistically significant differences between students' attitudes regarding the type of mobile device. The emergence of mobile learning technologies had a significant impact on the educational technology.

RESUMO

Na última década, surgiu um número significativo de iniciativas que visam a utilização de tecnologias e aplicações móveis para fins educacionais, no entanto, a implementação não tem sido totalmente eficaz devido a várias dificuldades. Neste trabalho, foram exploradas as atitudes de alunos e professores em relação ao uso da aprendizagem móvel em instituições de ensino superior. Foram coletadas amostras de alunos e professores de cinco universidades de Cajamarca, no Peru, nas quais foram aplicadas pesquisas que foram validadas por especialistas e a confiabilidade foi avaliada pelo alfa de Cronbach. Foi avaliado se existem diferenças estatisticamente significativas entre as atitudes dos alunos em relação à idade, sexo, especialidade e tipo de móvel utilizado. Da mesma forma, avaliou-se se existem diferenças estatisticamente significativas entre as atitudes dos professores em relação à idade, sexo, experiência universitária, nível de escolaridade e tipo de celular utilizado. Os testes t de Student e uma análise de variância unilateral (ANOVA) foram realizados para cada indicador considerado. Os resultados revelaram que existem diferenças estatisticamente significativas entre as atitudes dos alunos em relação ao tipo de dispositivo móvel. O surgimento de tecnologias de aprendizagem móvel teve um impacto significativo na tecnologia educacional. 


\section{Introducción}

Dado el avance vertiginoso de las tecnologías de información y comunicación siempre ha sido foco de interés la incorporación de estas herramientas en el ámbito educativo (Bernier, 2019). Esta revolución de las comunicaciones en la era digital trajo consigo un gran cambio de paradigma en el sector educativo (Machado y Díaz, 2018). La implementación de diversas estrategias desde el ámbito digital puede elevar la calidad en la educación ayudando a superar ciertas limitaciones que imponen las estrategias tradicionales de enseñanza y aprendizaje (Otero et al., 2019). Esta implementación no solo es en el ámbito estudiantil sino también en las competencias docentes que permitan un efectivo logro de las metas planteadas (González, 2016).

En el caso del aprendizaje de las matemáticas siempre se ha reflejado preocupación en el rendimiento general de los estudiantes, al respecto Medina, Fereira y Marzol (2018) reportaron que los factores personales de los estudiantes inciden medianamente en el bajo rendimiento de los estudiantes de Geometría por lo que recomiendan implementar nuevas estrategias que contribuyan a la motivación estudiantil en dirección a un mayor compromiso con las asignaturas.

La disponibilidad de teléfonos inteligentes (Smartphones) a precios asequibles ha llevado a un aumento en el uso de aplicaciones (Apps) para diversos aspectos de la vida, como la comunicación, los viajes, el entretenimiento, la productividad y el aprendizaje. En la última década, se han dado un número significativo de iniciativas que apuntan a utilizar tecnologías y aplicaciones móviles con fines educativos (Kearney, Burden y Rai, 2015; Bano et al., 2018; Romero, 2020).

En la literatura, los investigadores han definido el aprendizaje móvil desde diferentes perspectivas. Mcconatha, Praul y Lynch (2008) han definido el aprendizaje móvil como aquel que se emplea mediante el uso de pequeños dispositivos móviles informáticos. Esta definición incluye teléfonos inteligentes y pequeños dispositivos de mano. Además, Mirski y Abfalter (2004) definieron el aprendizaje móvil como un tema específico que está surgiendo del aprendizaje a distancia; mientras que Ardies, De Maeyer, Gijbels y van Keulen (2015) argumentaron que las actitudes hacia cualquier tecnología educativa podrían usarse para saber en qué medida los usuarios (estudiantes y educadores) tienen la ambición de usar la tecnología y si esto tiene impactos positivos o negativos en el medio ambiente. Barki y Hartwick (1994) han respaldado empíricamente que las actitudes de los usuarios conducen a las intenciones de uso y al usuario real del nuevo sistema.

Varias universidades de todo el mundo han implementado este enfoque para ofrecer el aprendizaje en cualquier momento y lugar de diferentes maneras. En Canadá College y San Francisco State University (SFSU) se implementó el modelo de Red de Aprendizaje Interactivo (ILN, por sus siglas en inglés) que involucra tanto tabletas como tecnología inalámbrica para evaluaciones previas y posteriores para determinar el rendimiento de los estudiantes (Enríquez, 2010). Erkollar y Oberer (2012) abordaron la integración del aprendizaje móvil con el módulo del Sistema de Información Geográfica (GIS) en un curso piloto dentro de una universidad turca donde cada estudiante recibió un dispositivo de tableta equipado con Google y Hangout Apps para facilitar la comunicación de los alumnos. Glackin, Rodenhiser y Herzog (2014) abordaron la integración de dispositivos móviles y libros electrónicos para aumentar la familiaridad de los estudiantes con la biblioteca digital. De Pablos, Tennyson y Lytras (2015) realizaron dos estudios en la Universidad Americana de Sharjah, para estudiantes de pregrado para examinar el uso de iPads durante un semestre en el curso de Matemáticas.

A pesar de que el aprendizaje móvil se ha estado implementando por diversas instituciones, es pertinente en el caso de la región de Cajamarca realizar un estudio de las actitudes de los estudiantes y docentes respecto a la implementación del aprendizaje móvil en las aulas. De modo que el objetivo de este estudio fue explorar las actitudes de los alumnos y docentes hacia el uso del aprendizaje móvil en las instituciones de educación superior con el fin de predecir su intención de utilizar la tecnología móvil en el contexto educativo. Por lo tanto, esta investigación busca responder las siguientes preguntas de investigación:

¿Existe alguna diferencia significativa entre las actitudes de los estudiantes hacia el uso del aprendizaje móvil con respecto al sexo, especialización, tipo de móvil?

¿Existe alguna diferencia significativa entre las actitudes de los docentes hacia el uso del aprendizaje móvil con respecto al sexo, nivel académico, experiencia, tipo de móvil? 


\section{Metodología}

Para este trabajo se realizó un estudio de tipo exploratorio centrado en conocer cómo los estudiantes y docentes de educación superior perciben la utilidad de los dispositivos móviles en el contexto educativo.

\section{Población y muestra}

La población del estudio constó de cinco instituciones de educación superior de la región de Cajamarca, Perú. Las universidades participantes fueron la Universidad Nacional de Cajamarca (UNC), Universidad Nacional de Chota (UNACH), Universidad Privada Antonio Guillermo Urrelo (UPAGU), Universidad de San Pedro (USP) y la Universidad Privada del Norte (UPN). Los datos se recopilaron en los meses de febrero y marzo del 2019 mediante dos encuestas: una para estudiantes y otra para docentes. La tabla 1 muestra los detalles de la muestra seleccionada:

Tabla 1. Participantes en el estudio

\begin{tabular}{|c|c|c|}
\hline Universidad & $\begin{array}{c}\text { Nro. de } \\
\text { Estudiantes }\end{array}$ & $\begin{array}{l}\text { Nro. de } \\
\text { docentes }\end{array}$ \\
\hline Universidad Nacional de Cajamarca (UNC) & 90 & 25 \\
\hline Universidad Nacional de Chota (UNACH) & 90 & 25 \\
\hline $\begin{array}{l}\text { Universidad Privada Antonio Guillermo } \\
\text { Urrelo (UPAGU) }\end{array}$ & 90 & 25 \\
\hline Universidad San Pedro (USP) & 90 & 25 \\
\hline Universidad Privada del Norte (UPN) & 90 & 25 \\
\hline Total & 450 & 125 \\
\hline ente: Elaboración de los autores & & \\
\hline
\end{tabular}

El estudio utilizó un enfoque de muestreo intencional donde las muestras extraídas para el caso de los estudiantes fueron de las especialidades: Educación, Contabilidad, Derecho e Ingeniería Ambiental. Los educadores también fueron seleccionados de diferentes rangos y experiencias académicas (Onwuegbuzie y Collins, 2007; Teddlie y Yu, 2007). Después de obtener la aprobación ética de las universidades mencionadas, los investigadores aplicaron el cuestionario presencial entregado personalmente en clase. Se informó a los participantes que su colaboración es totalmente voluntaria en el estudio y que sus respuestas son confidenciales.

\section{Instrumentos}

Las encuestas aplicadas tanto a los docentes y estudiantes constó de 28 ítems (preguntas). La primera sección estuvo conformada de ocho ítems donde se registró la información personal, datos demográficos del estudiante o docente. La segunda sección estuvo conformada por diez ítems que registraban la información del estudiante o docente respecto a la tecnología móvil usada. La tercera sección de la encuesta constó de diez ítems que evaluaron las actitudes hacia el uso del aprendizaje móvil. Estas encuestas fueron diseñadas siguiendo la escala tipo Likert de cinco puntos para medir los diez ítems relacionados a la actitud: totalmente de acuerdo (5), De acuerdo (4), Indeciso (3), En desacuerdo (2) y totalmente en desacuerdo (1). Las actitudes se han calculado combinando los diez ítems de la tercera sección de cada encuesta. 
Las dos encuestas han sido desarrolladas por los autores, sin embargo, algunos elementos se han adoptado de estudios relevantes para garantizar la validez del contenido (Liaw y Huang, 2012; Cavus, 2011; Alwraikat y Al Tokhaim, 2014) y garantizar su validez (Chang y Tung, 2008). Ambas hacían referencia al aprendizaje móvil, La primera a las actitudes de los estudiantes y la segunda, a las actitudes de los docentes. Las encuestas fueron sometidas a juicio de tres expertos de la Universidad de Cajamarca para verificar la claridad de las preguntas. Todas fueron aleatorizadas para evitar el sesgo debido a que la agrupación de preguntas puede influir una sobre la otra (Ardies et al., 2015).

\section{Análisis de los datos}

Se efectuó un previo análisis general mediante estadística descriptiva junto a un estudio factorial exploratorio para las preguntas relacionadas con la actitud en ambas encuestas utilizando el método de extracción de análisis de componentes principales con rotación Varimax. Las actitudes de los alumnos (P1-10 en la tabla 2) se cargaron en un solo factor (entre 0.787 y 0.891) y las actitudes de los docentes (P1-10 en la tabla 3) se cargaron en un solo factor (entre 0.691 y 0.881 ).

Se realizó una prueba de confiabilidad para las diez variables independientes que representan las actitudes calculando el alfa de Cronbach (Khaddage y Knezek, 2013; Zhang,). Dichos valores del alfa de Cronbach para las actitudes de los estudiantes fueron de 0.912 y 0.925 para los docentes, ambos mayores a 0.7.

Para evaluar si existe diferencias significativas entre las actitudes de los estudiantes y las otras variables se efectuaron pruebas t de Student y análisis de varianza unidireccional (Anova), todos los análisis estadísticos se efectuaron utilizando el programa SPSS ${ }^{\circledR}$ versión 25.

\section{Resultados}

Al analizar los datos personales y demográficos de los estudiantes, los resultados indicaron que el $58.2 \%$ de los estudiantes son mujeres y el 40.8 \% son varones. En cuanto a las edades, el $89.1 \%$ de los estudiantes tiene edades comprendidas entre 18 y 22 años. El 53.7\% de los estudiantes provenían de la especialidad de Educación, mientras que los estudiantes de Contabilidad, Derecho e Ingeniería Ambiental con 28\%, 11.4\% y $6.4 \%$, respectivamente.

Por otro lado, al analizar los datos personales y demográficos de los docentes, los resultados revelaron que el $70.1 \%$ de los educadores eran hombres, mientras que el resto mujeres. El $49.1 \%$ de los docentes poseen título de maestría, mientras que aquellos con doctorado y licenciatura fueron 33.6\% y $17.1 \%$, respectivamente. El $59 \%$ de los participantes estaban en el rango de instructor.

Al analizar la información en cuanto a los dispositivos móviles de los estudiantes los resultados mostraron que el $70.8 \%$ de estos solo poseen un Smartphone, además, el $28.8 \%$ poseen una tableta, mientras que solo el resto de los estudiantes no poseen ni Smartphone ni Tablet. De estos estudiantes, el 44.6\% están usando sus dispositivos móviles (smartphone o tablet) para navegar en la web, acceder a sus correos electrónicos y utilizar Apps para funciones específicas, mientras que el 15.8\% de estos estaban usando sus dispositivos móviles como herramientas para estudiar bien sea mediante búsquedas de información por la web, mensajería, así como también la descarga de Apps específicas que les sirva como herramientas complementarias en su aprendizaje. "WhatsApp" es la aplicación de mensajería más popular ya que es utilizada por el $86.7 \%$ de los estudiantes.

Al analizar las actitudes de los estudiantes hacia la tecnología móvil, se ha observado que el puntaje promedio total de las actitudes fue de 3.21 y el puntaje promedio de su percepción de utilidad al usar dispositivos móviles en sus estudios fue de 3.18, la percepción de estos en cuanto al papel en el apoyo a la comunicación con sus compañeros y docentes ha logrado el puntaje promedio más alto de 3.587. También, el puntaje promedio de la percepción de la superación personal y el desarrollo de sus procesos y habilidades de aprendizaje fue de 3.35 .

Al analizar la información de tecnología móvil en los docentes, los resultados han indicado que el 59.3\% de docentes poseen un Smartphone, el $41.2 \%$ posee un Smartphone y una tableta, y el $2.4 \%$ de ellos no tiene ninguno. El 58.9\% de los docentes usa sus dispositivos móviles para navegar en la web, acceder a sus correos electrónicos y utilizan Apps. De este grupo, solo el 18.4\% usa sus dispositivos móviles para el aprendizaje / educación en particular utilizan Apps para mejorar sus estrategias. El 79.8\% de los docentes indicaron que no estaban usando sus dispositivos móviles en la enseñanza. WhatsApp toma el porcentaje más alto con el $80.5 \%$ 
en comparación con Telegram (14.1\%) y SMS (2.1\%). El 65\% de los docentes indicaron que el uso diario de sus dispositivos móviles con fines educativos es inferior a dos horas.

Al analizar las actitudes de los docentes hacia la tecnología móvil, encontramos que el puntaje promedio total de las actitudes de los educadores fue de 3.53 y el puntaje promedio de su percepción de utilidad del uso de dispositivos móviles en el proceso educativo fue de 3.78. El puntaje promedio del rol de la tecnología móvil en el apoyo a la comunicación con otros colegas y estudiantes fue de 3.74, mientras que el puntaje promedio de la percepción de facilitar el acceso, la recuperación y el intercambio de información y materiales fue 4.12. El puntaje promedio de la percepción usando el aprendizaje móvil para los estudiantes fue de 3.32, y el puntaje promedio de la percepción de la superación personal y el desarrollo de sus procesos y habilidades laborales fue de 3.57 .

A continuación, se presentan los resultados que responden a las preguntas de investigación planteadas.

P: ¿Existe alguna diferencia significativa entre las actitudes de los estudiantes hacia el uso del aprendizaje móvil con respecto al sexo?

Se realizó una prueba $t$ de Student independiente para examinar si existe alguna diferencia significativa entre las actitudes de los estudiantes hacia el uso del aprendizaje móvil con respecto a su sexo. De acuerdo a la tabla 2, los valores medios para estudiantes masculinos y femeninos no indican diferencias significativas entre los estudiantes en cuanto a sus actitudes en términos de su género. El valor calculado de t fue de 1.289 y el nivel de significancia $p=0.378,(p>0.05)$. El resultado de esta pregunta podría atribuirse al hecho de que los estudiantes varones en la región están trabajando con estudiantes hembras en casi todas las actividades y ambos tienen suficiente manejo de la tecnología móvil. Por lo tanto, no se ha notado ninguna diferencia estadísticamente significativa.

P: ¿Existe alguna diferencia significativa entre las actitudes de los docentes hacia el uso del aprendizaje móvil con respecto al sexo?

Para examinar si hay alguna diferencia estadística significativa entre las actitudes de los docentes hacia el uso del aprendizaje móvil con respecto a su género se efectuó un análisis $t$ de Student. En la tabla 2 se muestran los resultados donde se indican que las puntuaciones medias para hombres y mujeres no implican diferencias significativas ( $p=0.412, p>0.05)$ entre docentes en sus actitudes con respecto a su género.

Tabla 2. Diferencias entre las actitudes de los estudiantes y docentes con respecto a su género

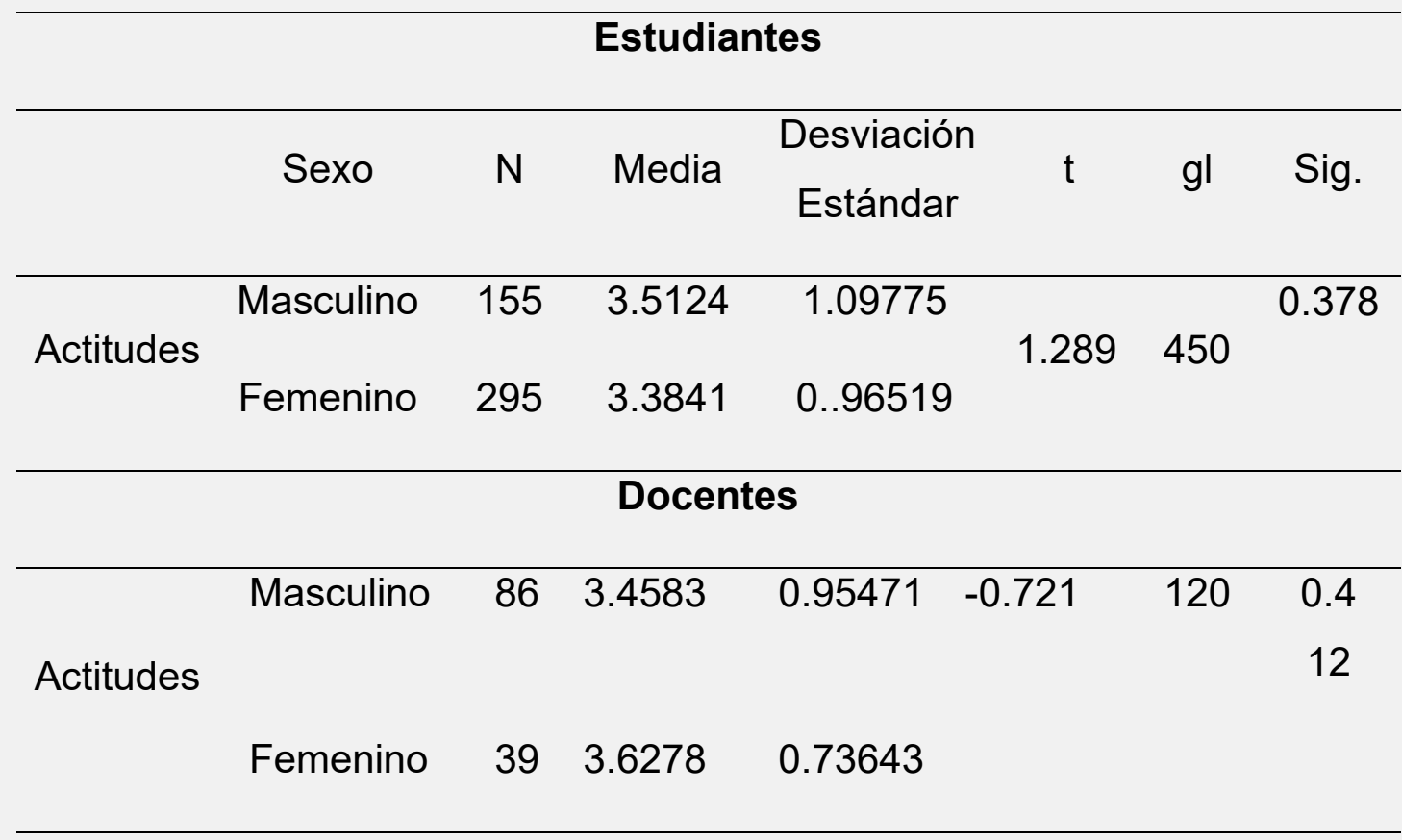

Fuente: Elaboración de los autores. 
P: ¿Existe alguna diferencia significativa entre las actitudes de los estudiantes hacia el uso del aprendizaje móvil con respecto a su especialización?

Se realizó un análisis de varianza unidireccional (Anova) para probar si hay alguna diferencia estadística significativa entre los valores medios. Como se muestra en la tabla 3, los resultados revelaron que no hay diferencias estadísticamente significativas $(p=0.914, p>0.05)$ entre las actitudes de los estudiantes con respecto a sus especialidades académicas. El resultado de esta pregunta de investigación podría referirse a la razón por la que casi todos los estudiantes estaban usando sus dispositivos móviles (teléfonos inteligentes / tabletas) para acceder a sus correos electrónicos, chatear en las redes sociales, compartir archivos a través de servicios en la nube. Por lo tanto, no se han reportado diferencias significativas.

P: ¿Existe alguna diferencia significativa entre las actitudes de los estudiantes hacia el uso del aprendizaje móvil con respecto al tipo de dispositivo móvil usado?

El análisis de varianza unidireccional (Anova) para examinar si hay alguna diferencia estadística significativa entre los valores medios se muestra en la tabla 3. Los resultados revelaron que existen diferencias estadísticamente significativas ( $p=0.047, p \leq 0.05$ ) entre las actitudes de los estudiantes con respecto al tipo de dispositivo móvil usado.

Para determinar dónde se producen las diferencias en los valores medios, se utilizó la prueba de Tukey para las comparaciones post-hoc. Los resultados indicaron que existen diferencias estadísticas entre las actitudes de los estudiantes con teléfonos inteligentes y aquellos con teléfonos inteligentes y tabletas, donde las diferencias están a favor de ambos dispositivos.

Tabla 3. Resultados de ANOVA para las actitudes de los estudiantes con respecto a su especialidad

\begin{tabular}{|c|c|c|c|c|c|c|}
\hline \multicolumn{7}{|c|}{ Con respecto a su especialidad } \\
\hline & $\begin{array}{l}\text { Suma de } \\
\text { cuadrados }\end{array}$ & & $\mathrm{gl}$ & $\begin{array}{c}\text { Error } \\
\text { cuadrátic } \\
\text { o medio }\end{array}$ & $\mathrm{F}$ & Sig. \\
\hline Intra Grupos & 0.479 & & 3 & 0.162 & 0.156 & 0.914 \\
\hline $\begin{array}{l}\text { Dentro de los } \\
\text { grupos }\end{array}$ & 393.244 & & 425 & 1.035 & & \\
\hline Total & 393.723 & & 428 & & & \\
\hline \multicolumn{7}{|c|}{ Con respecto al tipo de móvil utilizado } \\
\hline Intra Grupos & & 9.124 & 3 & 3.125 & 3.154 & 0.047 \\
\hline Dentro de los & & 395.654 & 425 & 1.204 & & \\
\hline Total & & 404.778 & 428 & & & \\
\hline
\end{tabular}

Fuente: elaboración de los autores

P: ¿Existe alguna diferencia significativa entre las actitudes de los docentes hacia el uso del aprendizaje móvil con respecto a su nivel académico?

El análisis de varianza unidireccional (Anova) para examinar si hay alguna diferencia estadística significativa entre los valores medios se muestran en las tablas 4 y 5 . Los resultados revelaron que no existen diferencias estadísticamente significativas ( $p=0.398, p>0.05)$ entre las actitudes de los docentes con respecto a su nive académico. 
Tabla 4. Desviación media y estándar para las actitudes de los docentes con respecto a su nivel y experiencia

\begin{tabular}{|c|c|c|c|}
\hline Nivel Académico & $\mathbf{N}$ & Media & $\begin{array}{c}\text { Desviación } \\
\text { Estándar }\end{array}$ \\
\hline Instructor & 53 & 3.4301 & 0.66275 \\
\hline Profesor Asistente & 31 & 3.4111 & 1.15358 \\
\hline Profesor Asociado & 23 & 4.1212 & 0.49748 \\
\hline $\begin{array}{l}\text { Profesor Dedicación } \\
\text { Exclusiva }\end{array}$ & 18 & 3.6700 & 0.12764 \\
\hline Total & 125 & 3.5148 & 0.71401 \\
\hline $\begin{array}{l}\text { Experiencia } \\
\text { Académica }\end{array}$ & $\mathbf{N}$ & Media & $\begin{array}{l}\text { Desviación } \\
\text { Estándar }\end{array}$ \\
\hline Menos de 5 años & 38 & 3.4121 & 0.75347 \\
\hline Entre 5 y 10 años & 12 & 3.8000 & 0.82877 \\
\hline Más de 10 años & 75 & 3.5175 & 0.84021 \\
\hline Total & 125 & 3.7361 & 0.86623 \\
\hline
\end{tabular}

Fuente: elaboración de los autores

P: ¿Existe alguna diferencia significativa entre las actitudes de los docentes hacia el aprendizaje móvil con respecto a su experiencia académica?

En las tablas 5 y 6 se muestran los valores de las medias y desviación estándar para el Anova. Los resultados indicaron que no hubo diferencias estadísticamente significativas ( $p=0.798, p>0.05)$ entre las actitudes de los educadores con respecto a su experiencia académica.

Tabla 5. Resultados de Anova para las actitudes de los docentes con respecto a su nivel y experiencia académica

\begin{tabular}{cccccc}
\hline \multicolumn{6}{c}{ Con respecto al nivel académico } \\
\hline & $\begin{array}{c}\text { Suma de } \\
\text { cuadrados }\end{array}$ & gl & $\begin{array}{c}\text { Error } \\
\text { cuadrático } \\
\text { medio }\end{array}$ & F & Sig. \\
\hline Intra Grupos & 2.115 & 3 & 0.668 & 0.950 & 0.398 \\
$\begin{array}{c}\text { Dentro de los } \\
\text { Total }\end{array}$ & 34.181 & 117 & 0.682 & & \\
\hline
\end{tabular}




\begin{tabular}{cccccc}
\hline \multicolumn{7}{c}{ Con respecto a la experiencia académica } \\
\hline & $\begin{array}{c}\text { Suma de } \\
\text { cuadrados }\end{array}$ & gl & $\begin{array}{c}\text { Error cuadrático } \\
\text { medio }\end{array}$ & F & Sig. \\
\hline Intra & 0.269 & 2 & 0.079 & 0.112 & 0.798 \\
Dentro de & 36.037 & 118 & 0.704 & & \\
Total & 36.306 & 120 & & & \\
\hline
\end{tabular}

Fuente: elaboración de los autores.

P: ¿Existe alguna diferencia significativa entre las actitudes de los docentes hacia el uso del aprendizaje móvil con respecto al tipo de dispositivo móvil usado?

Los valores de la media y las desviaciones estándar para el tipo de dispositivo móvil usado por los docentes se muestran en la tabla 6. Para examinar si existe alguna diferencia estadística significativa entre las puntuaciones medias se realizó un análisis de varianza de una vía (ANOVA) como se muestra en la Tabla referida. Los resultados indican que no hay diferencias estadísticamente significativas ( $p=0.447, p>0.05$ ) entre las actitudes de los educadores con respecto al tipo de dispositivo móvil utilizado.

Tabla 6. Actitudes de los docentes con respecto al tipo de dispositivo móvil

\begin{tabular}{|c|c|c|c|c|c|}
\hline \multicolumn{6}{|c|}{ Desviación media y estándar } \\
\hline Tipo de móvil & $\mathrm{N}$ & Media & & Desviaci & nn Estándar \\
\hline Smartphone & 70 & 35.812 & & & 4975 \\
\hline Tablet & 7 & 34.125 & & & - \\
\hline Ambos & 42 & 39.061 & & & 3385 \\
\hline Ninguno & 6 & 36.102 & & & 6569 \\
\hline Total & 125 & 37.370 & & & 2512 \\
\hline \multicolumn{6}{|c|}{ Resultados de ANOVA } \\
\hline & $\begin{array}{c}\text { Suma de } \\
\text { cuadrados }\end{array}$ & gl & $\begin{array}{c}\text { Error } \\
\text { cuadrático }\end{array}$ & $F$ & Sig. \\
\hline Intra Grupos & 2.450 & 3 & 0.888 & 1.262 & 0.447 \\
\hline Dentro de los grupos & 33.864 & 117 & 0.786 & & \\
\hline Total & 35.534 & 120 & & & \\
\hline
\end{tabular}

Fuente: elaboración de los autores

\section{Discusión de resultados}

Los resultados sobre las actitudes de los estudiantes con respecto a su género no reportan ninguna diferencia estadísticamente significativa, estas derivaciones coinciden con los estudios de Cavus (2011), Wang, Wu y Wang (2009), Uzunboylu, Cavus y Ercag (2009) y Yang (2012) quienes también encontraron que no hubo diferencias significativas entre las actitudes de los estudiantes hacia el uso del aprendizaje móvil con respecto a su género. Sin embargo, Taleb y Sohrabi (2012), Khaddage y Knezek (2013) en su investigación han indicado que existen diferencias significativas entre las actitudes de los estudiantes con respecto al género, donde las estudiantes fueron más positivas hacia el uso de teléfonos móviles que los varones. 
Por otra parte, con respecto a las actitudes de los estudiantes con respecto a sus especialidades académicas, tampoco se han reportado diferencias significativas, estos resultados contraponen los reportados por Khaddage y Knezek (2013) quienes indicaron que los estudiantes que poseen teléfonos inteligentes fueron más positivos hacia el aprendizaje móvil que aquellos que no poseen $(p<0.03)$. Y corroboran los del estudio de Taleb y Sohrabi (2012), los cuales revelaron que no hubo diferencias significativas entre las actitudes de los estudiantes hacia el uso del aprendizaje móvil en términos de sus especialidades académicas.

Con respecto a las actitudes de los docentes hacia el uso del aprendizaje móvil con respecto a su género, los resultados indican que las puntuaciones medias para hombres y mujeres no implican diferencias significativas $(p=0.412, p>0.05)$ entre docentes en sus actitudes con respecto a su género. Estos resultados son contradictorios con los de Alwraikat y Al Tokhaim (2014), quienes revelaron mediante el uso de una prueba t independiente que las actitudes de las instructoras eran más positivas hacia el móvil que los instructores. Además, Uzunboylu y Ozdamli (2011) indicaron que las actitudes de los instructores masculinos eran más positivas hacia el aprendizaje móvil que las instructoras.

Por otro lado, los resultados revelaron que no existen diferencias estadísticamente significativas $(p=0.398, p>$ 0.05 ) entre las actitudes de los docentes con respecto a su nivel académico, siendo estos totalmente diferentes con las derivaciones del estudio de Alwraikat y Al Tokhaim (2014) quienes indicaron mediante el uso de una prueba ANOVA que las actitudes de los instructores, es decir, jóvenes asistentes de enseñanza, eran más positivas hacia el aprendizaje móvil que el personal académico con rangos más altos. Asimismo, resaltaron que las actitudes de los docentes con 21 años de experiencia o más fueron más positivas hacia el aprendizaje móvil que los demás.

\section{Conclusiones}

La aparición de tecnologías de aprendizaje móvil tuvo un impacto significativo en la tecnología educativa. En este documento se ha destacado el estado del arte en el aprendizaje móvil con respecto a las actitudes de los estudiantes y docentes hacia el uso del aprendizaje móvil en las instituciones de educación superior. La principal contribución de este estudio fue explorar las actitudes de los estudiantes y educadores, lo que a su vez puede servir de base para instalar una infraestructura de aprendizaje móvil.

Los responsables de la toma de decisiones de esas instituciones académicas podrían tener en cuenta esas diferencias significativas en la implementación de sistemas de aprendizaje móvil en el futuro. El uso de tabletas y Smartphones mejorará las actitudes positivas de los estudiantes hacia el aprendizaje, lo que a su vez lleva a la intención de usar el aprendizaje móvil en la educación superior. Las diferencias en la edad podrían estimular a las autoridades en el diseño de un sistema especial de aprendizaje móvil que pueda adaptarse a todas las edades.

\section{Referencias bibliográficas}

Alwraikat, M., y Al Tokhaim, H (2014). Exploring the potential of mobile learning use among faculty members. International Journal of Interactive Mobile Technologies (IJIM). 8(3), 4-10 http://dx.doi.org/10.3991/ijim. v8i3.3682

Ardies, J., De Maeyer, S., Gijbels, D., y van K, H (2015). Students attitudes towards technology. International Journal of Technology and Design Education. 25, 43-65. https://doi.org/10.1007/s10798-014-9268-x

Bano, M., Zowghi, D., Kearney, M., Schuck, S., Aubuson, P (2018). Mobile learning for science and mathematics school education: A systematic review of empirical evidence. Computers \& Education. 121,30-58. https://doi.org/10.1007/s10798-014-9268-x

Barki, H., y Hartwick, J (1994). Measuring user participation, user involvement, and user attitude. MIS Quarterly. 18(1), 59-82, https://doi.org/ 10.2307/249610

Bernier, J (2019). Efectividad educativa del Facebook para el desarrollo de la comprensión lectora. Revista TELOS. 21 (2), 313-332. http://ojs.urbe.edu/index.php/telos/article/view/3113

Cavus, N. (2011). Investigating mobile devices and LMS integration in higher education: student perspectives. Procedia Computer Science. 3,1469-1474. https://doi.org/10.1016/j.procs.2011.01.033

Chang, Su CH., y Tung, Feng-Chen. (2008). An empirical investigation of students' behavioural intentions to use 
the online learning course websites. British Journal of Educational Technology, 39 (1),71-83. https:// doi.org/10.1111/j.1467-8535.2007.00742.x

De Pablos, P., Tennyson, R., y Lytras, M (2015). Assessing the role of Mobile technologies and distance learning in higher education. Reino Unido: Editorial IGI Global.

Enriquez, A (2010). Enhancing student performance using tablet computers. College Teaching. 58(3), 77-84. https://doi.org/10.1080/87567550903263859

Erkollar, A., \& Oberer, B (2012). Anytime. Everywhere. Mobile learning in higher education: creating a GIS course. Alemania: Computer applications for database, education, and ubiquitous computing Heidelberg

Glackin, B, Rodenhiser, R., y Herzog, B (2014). A library and the disciplines: a collaborative project assessing the impact of ebooks and mobile devices on student learning. The Journal of Academic Librarianship. 40(34), 299-306. https://doi.org/10.1016/j.acalib.2014.04.007

González, M (2016). Formación docente en competencias TIC para la mediación de aprendizajes en el Proyecto Canaima Educativo. Revista TELOS. 18 (3), 492-507. http://ojs.urbe.edu/index.php/telos/article/ view/741

Kearney, M., Burden, K., y Rai, T. (2015). Investigating teachers' adoption of signature mobile pedagogies. Computers \& Education. 80, 48-57. https://doi.org/10.1016/j.compedu.2014.08.009

Khaddage, F., y Knezek, G (2013). iLearn via mobile technology: a comparison of mobile learning attitudes among university students in two nations. In Advanced learning technologies (ICALT), 2013 IEEE 13th international conference on pp. 256e258. IEEE.

Liaw, Shu-Sheng., y Huang Hsiu-Mei (2012) A Case of Study of Investigating Users' Acceptance toward Mobile Learning. In: Gaol F. (eds) Recent Progress in Data Engineering and Internet Technology. Lecture Notes in Electrical Engineering, vol 157. Springer, Berlin: Heidelberg

Machado, J., y Díaz, G (2018). Recursos da web 2.0 no mestrado em ciências da educação no instituto superior de ciências de educação do Huambo. Revista TELOS. 20(1), 82-100 http://ojs.urbe.edu/index.php/telos/article/view/911

Medina, N., Fereira, J., y Marzol, R (2018) Factores personales que inciden en el bajo rendimiento académico de los estudiantes de geometría. Revista TELOS. 20 (1), 4-28. http://ojs.urbe.edu/index.php/telos/article/ view/899

Mcconatha, D., Praul, M., y Lynch, M (2008). Mobile learning in higher education: an empirical assessment of a new educational tool. The Turkish Online Journal of Educational Technology - TOJET. 7 (3), 15-21. Recuperado de: https://files.eric.ed.gov/fulltext/EJ1102943.pdf

Mirski, P., y Abfalter, D. (2004). Knowledge enhancement on site-guests' attitudes towards m-learning. In Proceeding of the information and communication technologies in tourism 2004, Cairo: Egypto.

Otero, A., Rivera, W., Pedraza, C., y Canay, J. R (2019). TIC para la educación: Sistema adaptativo basado en mecanismos de aprendizaje automático para la apropiación de tecnologías en estudiantes de educación media. Revista TELOS. 21 (3),526-543. www.doi.org/10.36390/telos213.03

Onwuegbuzie, A., y Collins, K (2007). A typology of mixed methods sampling designs in social science research. The Qualitative Report. 12 (2), 281-316. https://nsuworks.nova.edu/tqr/vol12/iss2/9

Romero, R. (2020). Depression and the meaning of life in university students in times of pandemic. International Journal of Educational Psychology, 9(3), 223-242. https://doi.org/10.17583/ijep.2020.6784

Taleb, Z., y Sohrabi, A (2012). Learning on the move: the use of mobile technology to support learning for university students. Procedia-Social and Behavioral Sciences. 69, 1102-1109 https://doi.org/10.1016/j. sbspro.2012.12.038.

Teddlie, Ch., y Yu, F (2007). Mixed methods sampling a typology with examples. Journal of Mixed Methods Research. 1(1), 77-100. https://doi.org/10.1177/1558689806292430 
Uzunboylu, H., Cavus, N., y Ercag, E. (2009). Using mobile learning to increase environmental awareness. Computers \& Education. 52(2), 381-389. https://doi.org/10.1016/j.compedu.2008.09.008

Uzunboylu, H., y Ozdamli, F (2011). Teacher perception for m-learning: scale development and teachers' perceptions. Journal of Computer Assisted Learning. 27(6), 544-556. https://doi.org/10.1111/j.13652729.2011.00415.x

Wang, Yin-Shun., Wu, Ming-Cheng., y Wang, Hsiu-Yuan (2009). Investigating the determinants and age and gender differences in the acceptance of mobile learning. British Journal of Educational Technology. 40 (1), 92-118. https://doi.org/10.1111/j.1467-8535.2007.00809.x

Yang, Shih-hsien (2012). Exploring college students' attitudes and self-efficacy of mobile learning. Turkish Online Journal of Educational Technology-TOJET. 11(4), 148-154. Recuperado de: http://files.eric.ed.gov/fulltext/EJ989264.pdf

Zhang, Xi., De Pablos, P., y Xu, Q (2014). Culture effects on the knowledge sharing in multi-national virtual classes: a mixed method. Computers in Human Behavior. 31. 491-498. https://doi.org/10.1016/j. chb.2013.04.021 